\title{
Coupling analysis between resonating metamaterial slabs using scattering parameters
}

\author{
Ugur Cem Hasar $^{\text {a,** }}$, Yunus Kaya ${ }^{\mathrm{b}}$, Mucahit Izginli ${ }^{\mathrm{c}}$, Hamdullah Ozturk ${ }^{\mathrm{a}}$, Mehmet Ertugrul d,e,f \\ Omar M. Ramahi ${ }^{g}$ \\ ${ }^{a}$ Department of Electrical and Electronics Engineering, Gaziantep University, Gaziantep, 27310, Turkey \\ ${ }^{\mathrm{b}}$ Department of Electricity and Energy, Bayburt University, Bayburt, 69000, Turkey \\ c Department of Electrical and Electronics Engineering, Hasan Kalyoncu University, Gaziantep 27410, Turkey \\ d Department of Electrical and Electronics Engineering, Ataturk University, Erzurum, 25240, Turkey \\ ${ }^{\mathrm{e}}$ Department of Electrical and Electronics Engineering, Universiti Putra Malaysia, 43400 UPM Serdang, Selangor, Malaysia \\ ${ }^{\mathrm{f}}$ Kyrgyz-Turkish Manas University, Faculty of Engineering, Djal, Bishkek 720038, Kyrgyzstan \\ $\mathrm{g}$ University of Waterloo, Department of Electrical and Computer Engineering, Waterloo, ON N2L 3G1, Canada
}

\section{A R T I C L E I N F O}

\section{Keywords:}

Metamaterials

Split-ring-resonator

Coupling

Signal flow graph

ABCD matrix

\begin{abstract}
A B S T R A C T
Metamaterial (MM) slabs in close proximity can couple with one another. This coupling can modify the electromagnetic response of the overall MM structure involving individual MM resonators. The signal flow graph technique along with the ABCD parameters are applied to quantitatively and qualitatively examine this coupling from simulated/measured scattering parameters of cascade connection of closely or largely separated MM slabs. S-parameter measurements of a MM slab constructed by split-ring-resonators were carried out at $\mathrm{X}$-band $(8.2-12.4 \mathrm{GHz})$ to validate our theoretical analysis.
\end{abstract}

\section{Introduction}

Metamaterial (MMs) are structures that demonstrate unusual electromagnetic characteristics, such as negative refractive index [1], which lead to many applications in different areas including perfect lenses [2], electromagnetic cloaks [3], absorbers [4], tunable devices [5], and sensors [6]. In order to get full benefit from these applications, electromagnetic responses of these materials need to be fully known. A variety of techniques were proposed such as the qualitative effective medium theory [7], field averaging [8], homogenization techniques [9], and techniques based on measured/simulated scattering (S-) parameters [10]. Among these techniques, the latter has attracted huge interest due to its implementation (i.e., ease of measurements) and feasibility of analyzing complex MM structures through 3D full-wave electromagnetic simulators [11-20].

MM structures are constructed, in general, by positioning periodically small resonating elements in the transverse plane. Such a construction inherently follows coupling phenomenon between these resonating elements [21]. In addition to this coupling, MM structures can interact with each other in the longitudinal direction [22]. These couplings (transverse and longitudinal) may modify not only the electromagnetic response of the individual MM resonating cells, but also the overall electromagnetic behavior of the entire MM structure [22].
As a consequence, the coupling between close MM resonators in the transverse as well as the longitudinal dimensions should be investigated to gain more understanding of electromagnetic response of $\mathrm{MM}$ structures.

Numerous studies have examined transverse and longitudinal couplings [5,21-31]. The studies in [22-24] examined the effect of both coupling types on electromagnetic response of split-ring-resonator (SRR) MMs with no additional gap. Because an additional gap can change the electromagnetic response of these MMs and thus gives additional flexibility for tailoring their electromagnetic behavior, the studies in $[21,25]$ analyzed, at microwave frequencies, the effect of multiple gaps on a change in the electromagnetic response of these slabs. Other studies focused on MM resonators at infrared and terahertz frequencies [26,30,31]. Some studies performed analyses of the tunability of MM structures by adjusting the degree of coupling between resonating MM elements [5,27-29]. Nonetheless, these studies did not examine the transverse and/or longitudinal coupling between MM resonating cells through S-parameters. For quantitative and qualitative studies of the coupling effects, the signal flow graph (SFG) technique and the transfer matrix (TM) technique have been applied based on S-parameters [32]. This paper examines the effect of longitudinal coupling on S-parameters of the coupling network. Sensitivity analysis

\footnotetext{
* Corresponding author.

E-mail address: uchasar@gantep.edu.tr (U.C. Hasar).
} 
will be used to evaluate the effect of any possible air gap between MM slabs. Experimental waveguide measurements are performed to validate the theoretical analysis.

\section{Theory}

In this section, a theoretical background is given for the SFG technique along with the TM technique [33] for quantitative and qualitative examination of longitudinal coupling between resonating elements.

\subsection{Two-Slabs in propagation direction}

Fig. 1(a) shows two slabs denoted by Slab-1 and Slab-2, cascaded in the wave propagation direction. To consider the effect of the coupling between these slabs due to their close proximity, a region (i.e., a coupling network) is assumed to be present between these slabs, as shown in Fig. 1(b). The slabs and coupling networks in Fig. 1(b) are modeled by the following S-parameters: $S_{11}^{(1)}, S_{12}^{(1)}, S_{21}^{(1)}$, and $S_{22}^{(1)}$ for the network of the first slab; $S_{11}^{(c)}, S_{12}^{(c)}, S_{21}^{(c)}$, and $S_{22}^{(c)}$ for the coupling network; and $S_{11}^{(2)}, S_{12}^{(2)}, S_{21}^{(2)}$, and $S_{22}^{(2)}$ for the network of the second slab.

In obtaining the overall response of cascaded networks, the SFG technique is applied. This technique is useful once the individual elements are modeled by S-parameters [33]. Application of this technique to the representation in Fig. 1(b) results in the following forward and backward S-parameters [34]

$S_{11}^{3 T}=S_{11}^{2 T}+\frac{S_{21}^{2 T} S_{12}^{2 T} S_{11}^{(2)}}{1-S_{11}^{(2)} S_{22}^{2 T}}, \quad S_{21}^{3 T}=\frac{S_{21}^{(2 T)} S_{21}^{(2)}}{1-S_{11}^{(2)} S_{22}^{2 T}}$,
$S_{22}^{3 T}=S_{22}^{(2)}+\frac{S_{21}^{(2)} S_{12}^{(2)} S_{22}^{2 T}}{1-S_{11}^{(2)} S_{22}^{2 T}}, \quad S_{12}^{3 T}=\frac{S_{12}^{2 T} S_{12}^{(2)}}{1-S_{11}^{(2)} S_{22}^{2 T}}$,

where

$S_{11}^{2 T}=S_{11}^{(1)}+\frac{S_{21}^{(1)} S_{12}^{(1)} S_{11}^{(c)}}{1-S_{11}^{(c)} S_{22}^{(1)}}, \quad S_{21}^{2 T}=\frac{S_{21}^{(1)} S_{21}^{(c)}}{1-S_{11}^{(c)} S_{22}^{(1)}}$,

$S_{22}^{2 T}=S_{22}^{(c)}+\frac{S_{21}^{(c)} S_{12}^{(c)} S_{22}^{(1)}}{1-S_{11}^{(c)} S_{22}^{(1)}}, \quad S_{12}^{2 T}=\frac{S_{12}^{(1)} S_{12}^{(c)}}{1-S_{11}^{(c)} S_{22}^{(1)}}$.

Here, the superscript ' $2 \mathrm{~T}$ ' denotes the result of cascaded networks of the Slab-1 and the Coupling network, whereas the superscript '3T' shows the full response of the structure in Fig. 1(b). It is seen from (1) and

(2) that the coupling network affects the overall response.

On the other hand, the TM technique (or the ABCD parameters) can be applied to examine the overall response as well as the coupling between cascaded MM slabs [35,36], as shown in Fig. 1(c). Mathematically, it is possible to write

$$
\left[\begin{array}{ll}
A_{w} & B_{w} \\
C_{w} & D_{w}
\end{array}\right]=\left[\begin{array}{ll}
A_{1} & B_{1} \\
C_{1} & D_{1}
\end{array}\right]\left[\begin{array}{ll}
A_{c} & B_{c} \\
C_{c} & D_{c}
\end{array}\right]\left[\begin{array}{ll}
A_{2} & B_{2} \\
C_{2} & D_{2}
\end{array}\right],
$$

where the letters ' $w$ ', ' 1 ', ' $c$ ', and ' 2 ' designate the cascaded configuration in Fig. 1(a) (with coupling), the left slab, the coupling section, and the right slab, respectively. ABCD parameters' correspondence of each network can be obtained from S-parameters [37]

$$
\begin{aligned}
& A=\left[\left(1+S_{11}\right)\left(1-S_{22}\right)+S_{21} S_{12}\right] / 2 S_{21}, \\
& B=\left[\left(1+S_{11}\right)\left(1+S_{22}\right)-S_{21} S_{12}\right] / 2 S_{21}, \\
& C=\left[\left(1-S_{11}\right)\left(1-S_{22}\right)-S_{21} S_{12}\right] / 2 S_{21}, \\
& D=\left[\left(1-S_{11}\right)\left(1+S_{22}\right)+S_{21} S_{12}\right] / 2 S_{21},
\end{aligned}
$$

From (3), the ABCD parameters for the coupling section can be obtained as

$$
\left[\begin{array}{ll}
A_{c} & B_{c} \\
C_{c} & D_{c}
\end{array}\right]=\left[\begin{array}{ll}
A_{1} & B_{1} \\
C_{1} & D_{1}
\end{array}\right]^{-1}\left[\begin{array}{ll}
A_{w} & B_{w} \\
C_{w} & D_{w}
\end{array}\right]\left[\begin{array}{ll}
A_{2} & B_{2} \\
C_{2} & D_{2}
\end{array}\right]^{-1}
$$

Here, $\star^{-1}$ denotes the inverse of the matrix $\star$. After evaluating the ABCD parameters of the coupling network from (5), the S-parameters of this network can determined

$S_{11}^{(c)}=\frac{A_{c}+B_{c}-C_{c}-D_{c}}{A_{c}+B_{c}+C_{c}+D_{c}}$,

$S_{12}^{(c)}=\frac{2\left(A_{c} D_{c}-B_{c} C_{c}\right)}{A_{c}+B_{c}+C_{c}+D_{c}}$,

$S_{21}^{(c)}=\frac{2}{A_{c}+B_{c}+C_{c}+D_{c}}$,

$S_{22}^{(c)}=\frac{-A_{c}+B_{c}-C_{c}+D_{c}}{A_{c}+B_{c}+C_{c}+D_{c}}$.

\subsection{Multiple-Slabs in propagation direction}

The analysis given in the previous subsection is applicable only for two slabs positioned in the propagation direction. However, this analysis can be extended to a composite structure involving multiple slabs ( $p=1,2, \ldots, N$ where $N$ denotes the total number of slabs) in the direction of propagation after exercising the following two steps. First, the ABCD parameters ( $A_{\text {before }}, B_{\text {before }}, C_{\text {before }}, D_{\text {before }}, A_{\text {after }}, B_{\text {after }}$, $C_{\text {after }}$, and $\left.D_{\text {after }}\right)$ of the slabs before $(p=1,2, \ldots, k)$ and after $(p=$ $k, k+1, \ldots, N)$ the interface of slabs $k$ and $k+1$, in the order the slabs are positioned, within which the coupling network is examined, are calculated using the simulated/measured S-parameters of the slabs using (4a)-(4d). Here, $k=1$, or $2, \cdots$, or $N-1$. Second, coupling Sparameters $S_{11 k}^{(c)}, S_{21 k}^{(c)}, S_{12 k}^{(c)}$, and $S_{22 k}^{(c)}$ at the slab interface $(k, k+1)$ can be computed from

$S_{11 k}^{(c)}=\frac{A_{c k}+B_{c k}-C_{c k}-D_{c k}}{A_{c k}+B_{c k}+C_{c k}+D_{c k}}$

$S_{12 k}^{(c)}=\frac{2\left(A_{c k} D_{c k}-B_{c k} C_{c k}\right)}{A_{c k}+B_{c k}+C_{c k}+D_{c k}}$

$S_{21 k}^{(c)}=\frac{2}{A_{c k}+B_{c k}+C_{c k}+D_{c k}}$,

$S_{22 k}^{(c)}=\frac{-A_{c k}+B_{c k}-C_{c k}+D_{c k}}{A_{c k}+B_{c k}+C_{c k}+D_{c k}}$

where

$\left[\begin{array}{ll}A_{c k} & B_{c k} \\ C_{c k} & D_{c k}\end{array}\right]=\left[\begin{array}{ll}A_{\text {before }} & B_{\text {before }} \\ C_{\text {before }} & D_{\text {before }}\end{array}\right]^{-1}\left[\begin{array}{ll}A_{w} & B_{w} \\ C_{w} & D_{w}\end{array}\right]\left[\begin{array}{ll}A_{\text {after }} & B_{\text {after }} \\ C_{\text {after }} & D_{\text {after }}\end{array}\right]^{-1}$

\section{Computational analysis}

The entire analysis in this section is separated into two parts. In the first part, the theoretical background given in Section 2 is validated by considering a cascaded network consisting of two identical conventional materials. In the second part, two identical MM slabs are cascaded to examine the ABCD and S-parameters of the coupling network.

\subsection{Electrical properties and geometrical details of conventional materials and MM slabs}

Two different types of samples were used for validation of our theoretical analysis. The first type is a typical conventional isotropic polyethylene (PE) sample with complex relative permittivity $\varepsilon_{r}=2.25-$ $i 4.4444 \times 10^{-5}$ and physical dimensions of $3.85 \times 10.16 \times 22.86 \mathrm{~mm}^{3}$. It exactly fits to an X-band $(8.2-12.4 \mathrm{GHz}, a=22.86 \mathrm{~mm}$ and $b=10.16$ $\mathrm{mm}$ ) rectangular metallic waveguide. For a second sample type, two different MM slabs constructed by C-shaped square SRRs are considered in our study due to their ease of construction and widespread usage [1,22], although coupling between adjacent MM slabs involving various resonators such as $\pi$-shaped [38], U-shaped [39], octagonshaped [40], and $\Lambda$-shaped [41] resonators could as well be examined by our proposed method. In construction of these MM slabs with Cshaped square SRRs, the MM cell in Fig. 2(a) was considered. It is 

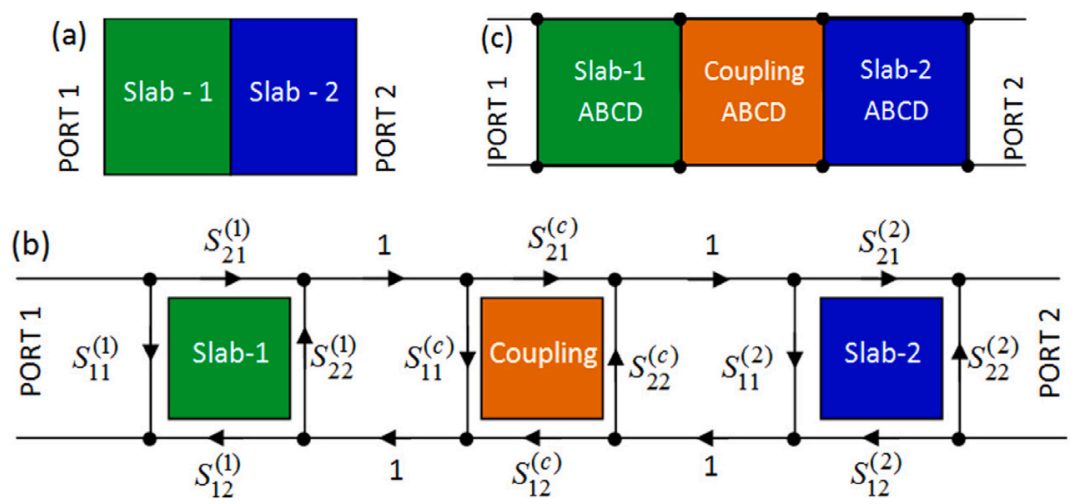

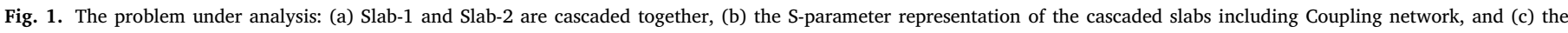
$A B C D$ parameter correspondence of representation in Fig. 1(b).
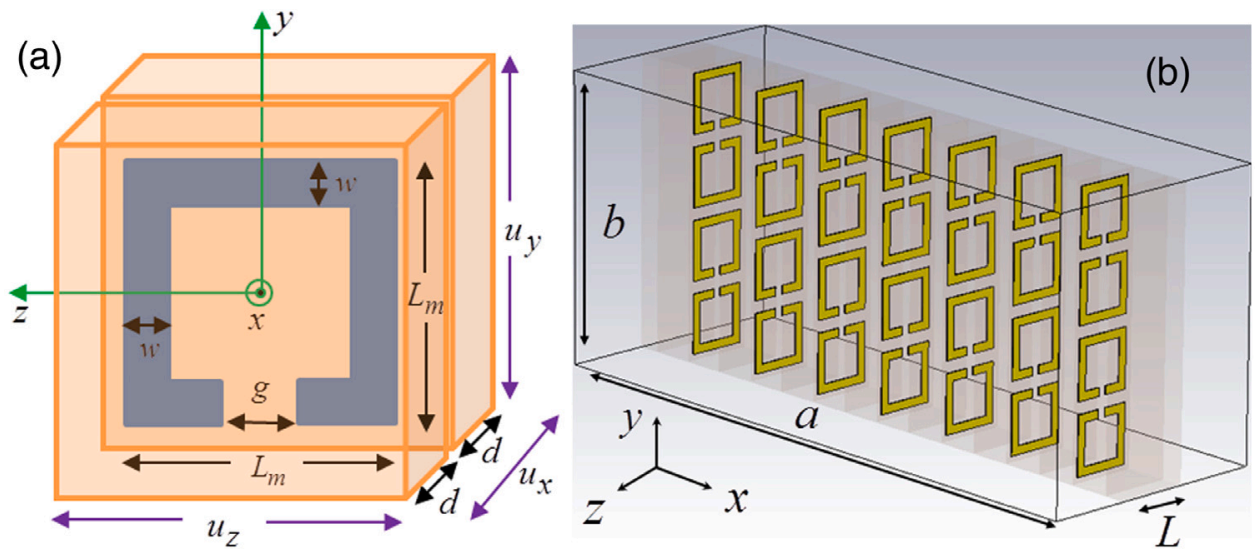

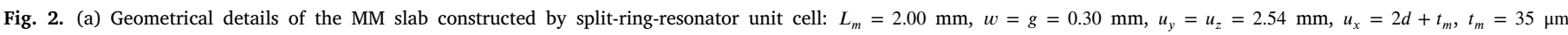

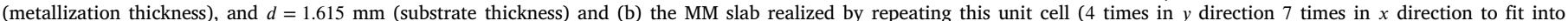
an X-band waveguide cross section $(a=22.86 \mathrm{~mm}$ and $b=10.16 \mathrm{~mm})$ ).

assumed to be made by a SRR pattern (copper with conductivity $\sigma=$ $5.8 \times 10^{7} \mathrm{~S} / \mathrm{m}$ and thickness $t_{m}=35 \mu \mathrm{m}$ ) sandwiched between two identical FR4 dielectric substrates which have a thickness of $d=1.615$ $\mathrm{mm}$, a length of $u_{z}=2.54 \mathrm{~mm}$, a height of $u_{y}=2.54 \mathrm{~mm}$, and a relative complex permittivity of $\varepsilon_{s}=4.3\left(1-i \delta_{e}\right)$ where the loss tangent is $\delta_{e}=0.025$ or 0.005 . We selected this substrate due to its availability in our laboratory for comparison of simulation results with measurement results. Similar to the usage of various forms of resonators, substrates with different electromagnetic properties (F4B, AD 25N, AD 450, and $\mathrm{AD} 600$ ) could as well be chosen in the analysis of coupling between nearby MM slabs by our proposed analysis. The geometrical details of a SRR pattern are presented in Fig. 2(a): metallization length $L_{m}=2.00$ $\mathrm{mm}$, width $w=0.30 \mathrm{~mm}$, and gap $g=0.30 \mathrm{~mm}$.

As discussed in Section 2.2, our proposed method is applicable for a composite MM structure having $N$ number of MM slabs in propagation direction. However, for simplicity, we only consider two different types of MM slabs. The first type MM slab demonstrated in Fig. 2(b) is realized by repeating 7 times the unit cell in the $x$ direction and 4 times in the $y$ direction (after reversing). The periodicities of the first MM slab in $x$ and $y$ directions are $u_{x}=3.27 \mathrm{~mm}$ and $u_{y}=2.54 \mathrm{~mm}$, both of which are much less than the free-space wavelength $(30 \mathrm{~mm})$ corresponding to $f=10 \mathrm{GHz}$ [42]. This shows that $a=7 u_{x}$ and $b=4 u_{y}$, meaning that the first MM slab fills the whole cross section of the X-band waveguide. The second MM slab is identical to the first one except for 11 times repetition of the unit cell in the $x$ direction (the thickness of the FR4 substrate is $1.02 \mathrm{~mm}$ ). The periodicities of the second MM slab in $x$ and $y$ directions are $u_{x}=2.078 \mathrm{~mm}$ and $u_{y}=2.54 \mathrm{~mm}$. These MM slabs are referred to the $\mathrm{MM}_{7}$ slab and the $\mathrm{MM}_{11}$ slab in the remaining manuscript. While the $\mathrm{MM}_{7}$ slab is selected to compare calculated and measured $S_{11}^{3 T}, S_{21}^{3 T}, S_{12}^{3 T}$, and $S_{22}^{3 T}$ (Section 5.3), there is no compelling reason for selecting the $\mathrm{MM}_{11}$ slab except for its different periodicity in the transverse plane ( $x y$ plane) with respect to the $\mathrm{MM}_{7}$ slab.

\subsection{Simulation details}

The full three-dimensional simulation software Computer Simulation Technology (CST) Microwave Studio was used to obtain the S-parameters of different configurations. Plane wave propagation and guided wave propagation through a rectangular waveguide could be equally applied to examine the coupling between nearby MM slabs by our methodology. However, in our present study, we preferred guidedwave propagation to plane wave propagation, because free-space measurements are hard to implement than guided-wave measurements due to the requirement of elimination of diffraction effects (wider sample dimension in transverse plane) and undesired reflections from ground or nearby objects $[43,44]$. Then, electric wall $\left(E_{t}=0\right)$ boundary conditions were applied at $x=\mp a / 2$ and at $y=\mp b / 2$ to realize waveguide metallic walls over the $x y$ plane. Two waveguide ports, $10 \mathrm{~mm}$ away from the surface of the MM slabs, were located at the $x y$ planes. Such boundary conditions and waveguide ports were used to simulate X-band (8.2-12.4 GHz) metallic waveguide structure operated at its dominant $\mathrm{TE}_{10}$ mode. Adaptive mesh property of CST Microwave Studio was functioned to optimize the meshing.

\subsection{Simulation results}

\subsubsection{Validation}

Cascade connection of two identical PE samples was considered for validation. Toward this end, S-parameters of each individual sample 

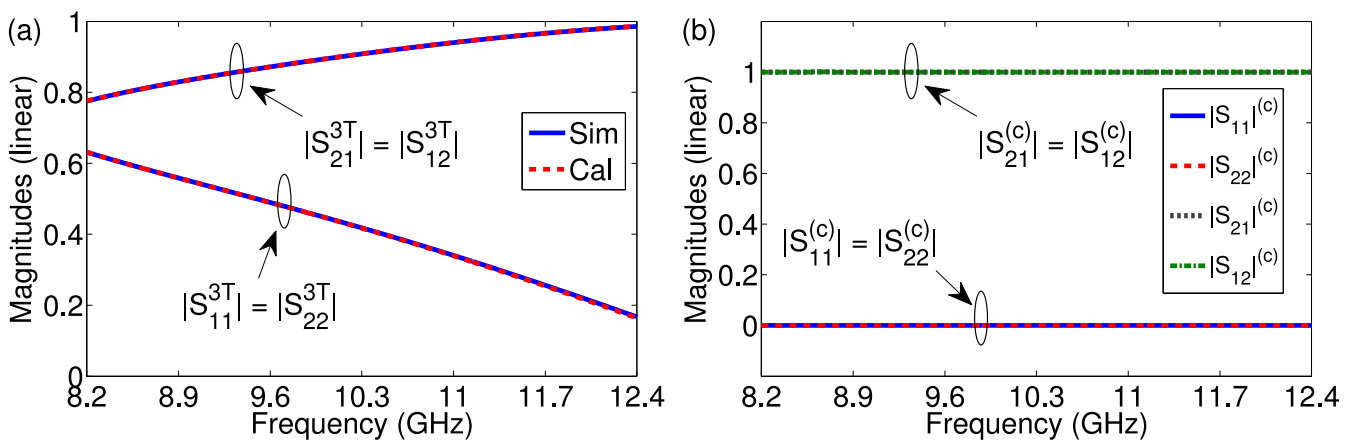

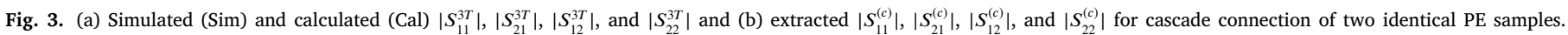
The conditions of $S_{11}^{(c)}=S_{22}^{(c)}=0$ and $S_{21}^{(c)}=S_{12}^{(c)}=1$ were considered. Reprinted with the permission of [32].

were first simulated and recorded as $S_{11}^{(1)}, S_{21}^{(1)}, S_{12}^{(1)}$, and $S_{22}^{(1)}$ for the first sample and $S_{11}^{(2)}, S_{21}^{(2)}, S_{12}^{(2)}$, and $S_{22}^{(2)}$ for the second sample. Next, S-parameters of cascade connection of these samples were simulated and recorded as $S_{11}^{3 T}, S_{21}^{3 T}, S_{12}^{3 T}$, and $S_{22}^{3 T}$. Then, $S_{11}^{3 T}, S_{21}^{3 T}, S_{12}^{3 T}$, and $S_{22}^{3 T}$ were determined from (1) and (2) using simulated S-parameters of the first and second samples under the assumption of no coupling; that is, $S_{11}^{(c)}=S_{22}^{(c)}=0$ and $S_{21}^{(c)}=S_{12}^{(c)}=1$. The validity of this assumption will be discussed shortly. Then, $A_{w}, B_{w}, C_{w}$, and $D_{w}$ parameters of the cascade connection were calculated from the simulated $S_{11}^{3 T}, S_{21}^{3 T}$, $S_{12}^{3 T}$, and $S_{22}^{3 T}$ using (4a)-(4d). The same procedure was repeated for the first and second PE samples. Finally, $S_{11}^{(c)}, S_{22}^{(c)}, S_{21}^{(c)}$, and $S_{12}^{(c)}$ parameters were evaluated from (6a)-(6d).

Fig. 3(a) exhibits simulated and calculated magnitudes $\left|S_{11}^{3 T}\right|,\left|S_{21}^{3 T}\right|$, $\left|S_{12}^{3 T}\right|$, and $\left|S_{22}^{3 T}\right|$ for the cascade connection of two identical PE samples under $S_{11}^{(c)}=S_{22}^{(c)}=0$ and $S_{21}^{(c)}=S_{12}^{(c)}=1$. Besides, Fig. 3(b) shows determined $\left|S_{11}^{(c)}\right|,\left|S_{21}^{(c)}\right|,\left|S_{12}^{(c)}\right|$, and $\left|S_{22}^{(c)}\right|$ terms using (5). For brevity, the phases of S-parameters are not given. The following remarks are noted from the dependencies in Fig. 3(a) and (b). First, because the PE sample material is symmetric and reciprocal, $\left|S_{11}^{3 T}\right|=\left|S_{22}^{3 T}\right|$ and $\left|S_{21}^{3 T}\right|=\left|S_{12}^{3 T}\right|$ over the full band. Second, simulated and calculated $\left|S_{11}^{3 T}\right|$ and $\left|S_{21}^{3 T}\right|$ are individually identical, meaning that, as expected, there is no coupling between PE samples (passive material). Third, extracted $S_{11}^{(c)}=S_{22}^{(c)}=0$ and $S_{21}^{(c)}=S_{12}^{(c)}=1$ results are in good agreement with our aforementioned assumption.

\subsubsection{Results of coupling between MM slabs}

Figs. 4 and 5 demonstrate the effect of substrate loss (solid curve for $\delta_{s}=0.025$ and dashed curve for $\delta_{s}=0.005$ ) on simulated Sparameters of the $\mathrm{MM}_{7}$ and $\mathrm{MM}_{11}$ slabs with length $2.54 \mathrm{~mm}$. The following points are noted from the dependencies in Figs. 4 and 5. First, as seen from Figs. 4(a) and 5(a), the transmission S-parameter reaches minimum value around $10.46 \mathrm{GHz}$ and $10.35 \mathrm{GHz}$ (degenerate resonance frequencies) for the $\mathrm{MM}_{7}$ slab $\left(\delta_{s}=0.005\right)$ and the $\mathrm{MM}_{11}$ slab $\left(\delta_{s}=0.005\right)$, respectively. Second, these resonance frequencies are split into two individual resonance frequencies of 9.565 and $10.464 \mathrm{GHz}$ for the cascade connection of the $\mathrm{MM}_{7}$ slabs, and 9.091 and $10.422 \mathrm{GHz}$ for the cascade connection of the $\mathrm{MM}_{11}$ slabs, due to coupling in the propagation direction ( $z$ direction) $[5,21,22,37]$. Third, the substrate loss broadens the band of resonance frequencies. Fourth, a decrease in the transverse $x$ dimension (from the $\mathrm{MM}_{7}$ slab to the $\mathrm{MM}_{11}$ slab) leads to a decrease in the resonance frequencies, which, we think, is due to a change in effective inductance and capacitance of the $\mathrm{MM}$ slab. For a dominant mode $\left(\mathrm{TE}_{10}\right)$ propagation inside the $\mathrm{X}$-band waveguide, the electric field will have one component in the $y$ direction and the magnetic field will have two components in the $x$ and $z$ directions. Therefore, whereas there is no electric coupling (electric field is directed over the slit axis), there is a magnetic coupling because the transverse magnetic field in the $x$-direction is perpendicular to the SRR plane $[5,22]$, and this magnetic coupling is more pronounced than the magnetic coupling created by longitudinal magnetic field after the SRR is rotated in the $y$-direction by $90^{\circ}$ (for this reason, in this study we use this dominant magnetic coupling) [22]. For the dominant magnetic coupling (magnetic excitation), symmetrical surface currents will flow over the SRRs according to the $x$ axis [5], which will definitely be affected by any additional gap over the SRR. These surface currents will accumulate charges of the same polarity at the corresponding gaps of the adjacent SRRs in the $x$ axis, producing negligible change in the overall capacitance. However, magnetic excitation will produce a significantly increased mutual inductance due to a decrease in the gap between corresponding SRRs in the $x$ axis, which in turn augment the overall inductance. Consequently, a downshift in the resonance frequency is observed for a decrease in the space between the SRRs in the $x$ direction.

$\left|S_{11}^{3 T}\right|,\left|S_{21}^{3 T}\right|,\left|S_{12}^{3 T}\right|$, and $\left|S_{22}^{3 T}\right|$ were then calculated for a cascade connection of both slabs. For example, Fig. 6(a) and (b) show simulated and calculated $\left|S_{11}^{3 T}\right|,\left|S_{21}^{3 T}\right|,\left|S_{12}^{3 T}\right|$, and $\left|S_{22}^{3 T}\right|$ for consecutive connection of two $\mathrm{MM}_{7}$ slabs $\left(\delta_{s}=0.025\right)$ and of two $\mathrm{MM}_{11}$ slabs $\left(\delta_{s}=0.025\right)$. In the analysis, $S_{11}^{(c)}=S_{22}^{(c)}=0$ and $S_{21}^{(c)}=S_{12}^{(c)}=1$ were set. The dependencies in Fig. 6(a) and (b) show a disagreement between simulated and calculated $\left|S_{11}^{3 T}\right|,\left|S_{21}^{3 T}\right|,\left|S_{12}^{3 T}\right|$, and $\left|S_{22}^{3 T}\right|$ for both cascaded MM slabs, mainly around the resonance region $\cong 10.3 \mathrm{GHz}$ ). This directly suggests that the coupling between the MM slabs created the observed deviation. To quantitatively evaluate the degree of coupling, $\left|S_{11}^{(c)}\right|,\left|S_{21}^{(c)}\right|,\left|S_{12}^{(c)}\right|$, and $\left|S_{22}^{(c)}\right|$ for both cascaded MM slabs were determined as shown in Figs. 7 (a) and 7(b), respectively. It is observed from Fig. 7 that $\left|S_{21}^{(c)}\right|$ and $\left|S_{12}^{(c)}\right|$ values pass over the unity value along the resonance regions of both cascaded MM slabs while the $\left|S_{11}^{(c)}\right|$ and $\left|S_{22}^{(c)}\right|$ values show rapid variation along these regions. This clearly indicates the energy transfer between MM slabs in the direction of propagation (the $z$ axis) [33].

To further examine the coupling or interference between closely or largely separated MM slabs in the direction of propagation, the magnitudes of the magnetic fields, as shown Fig. 8(a) and (b), at $10.485 \mathrm{GHz}$ for the cascaded $\mathrm{MM}_{7}$ slabs $\left(\delta_{s}=0.025\right)$ and at $10.371 \mathrm{GHz}$ for the cascaded $\mathrm{MM}_{11}$ slabs $\left(\delta_{s}=0.025\right)$ were analyzed. These figures illustrate spatial variation of magnetic fields between $2 \times 2$ SRR cells in $y$ and $z$ directions, with maximum and minimum levels represented by red and blue colors, respectively. Fig. 8(a) and (b) illustrate that only one cell pair is active in the direction of propagation [21]. An earlier study discussed in detail that different amplitudes and phases will be present if the excitation of the pairs is nonequal, meaning no symmetric or anti-symmetric excitation [21].

Our approach was also employed to monitor the effect of distance between the MM slabs on the coupling mechanism. This distance is assumed to be filled by FR4 material across the cross section of the X-band waveguide. Fig. 9(a) and (b) show the simulated and calculated $\left|S_{11}^{3 T}\right|,\left|S_{21}^{3 T}\right|,\left|S_{12}^{3 T}\right|$, and $\left|S_{22}^{3 T}\right|$ for cascaded two $\mathrm{MM}_{7}$ slabs $\left(\delta_{s}=0.025\right)$ and for cascaded two $\mathrm{MM}_{11}$ slabs $\left(\delta_{s}=0.025\right)$ when there is a $12 \mathrm{~mm}$ distance between these MM slabs. It is assumed that $S_{11}^{(c)}=S_{22}^{(c)}=0$ 

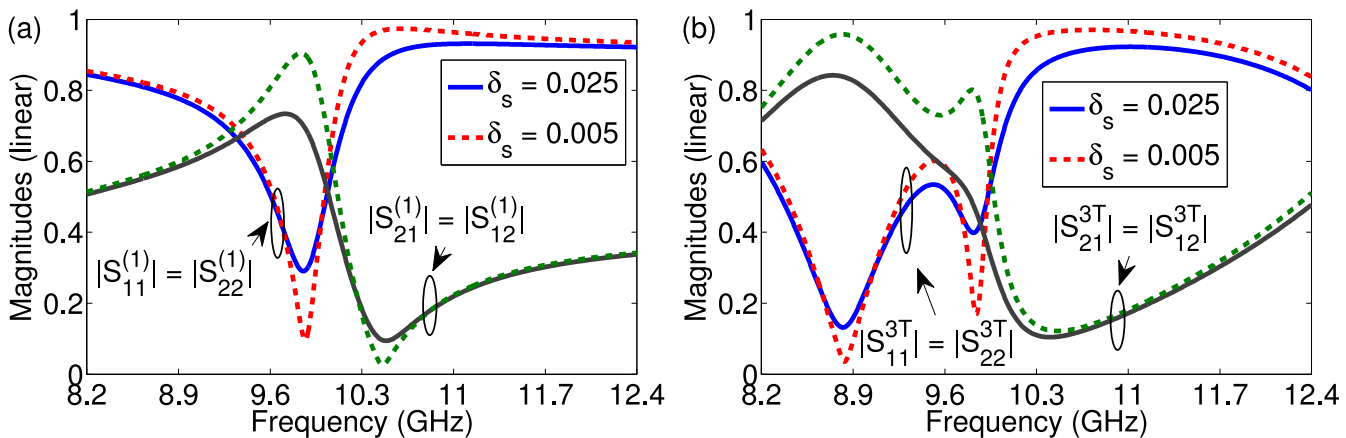

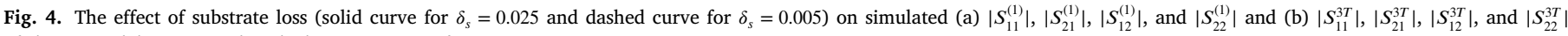
of the $\mathrm{MM}_{7}$ slab. Reprinted with the permission of [32].
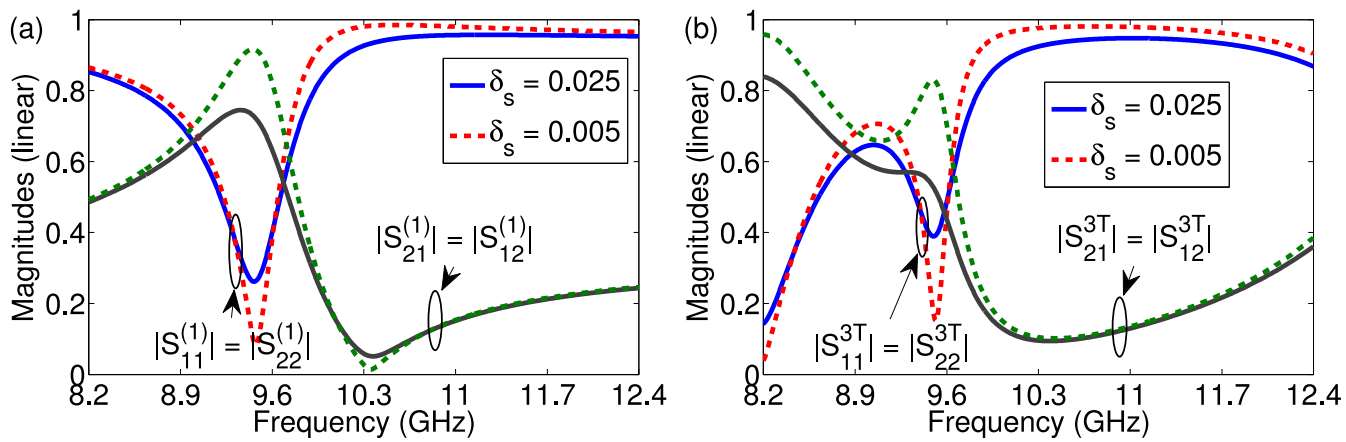

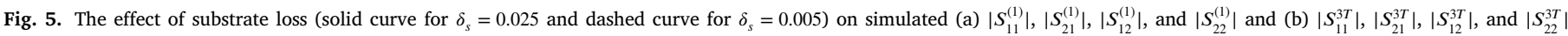
of the $\mathrm{MM}_{11}$ slab.
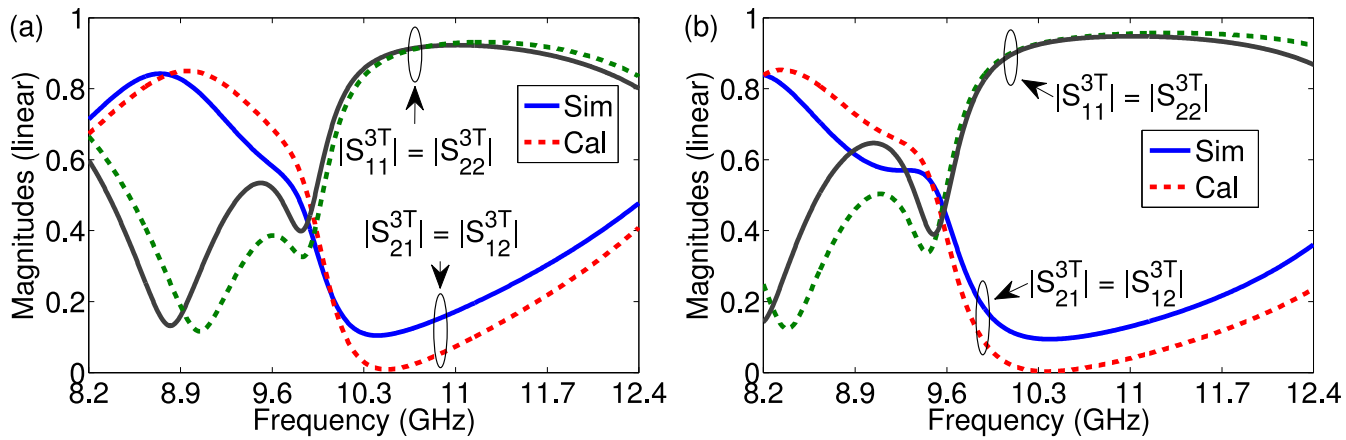

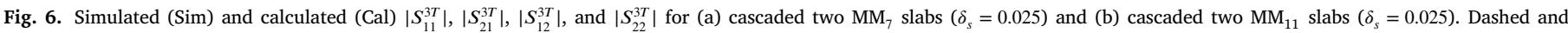
solid curves denote, respectively, the calculated and simulated dependencies. In the analysis, $S_{11}^{(c)}=S_{22}^{(c)}=0$ and $S_{21}^{(c)}=S_{12}^{(c)}=1$.
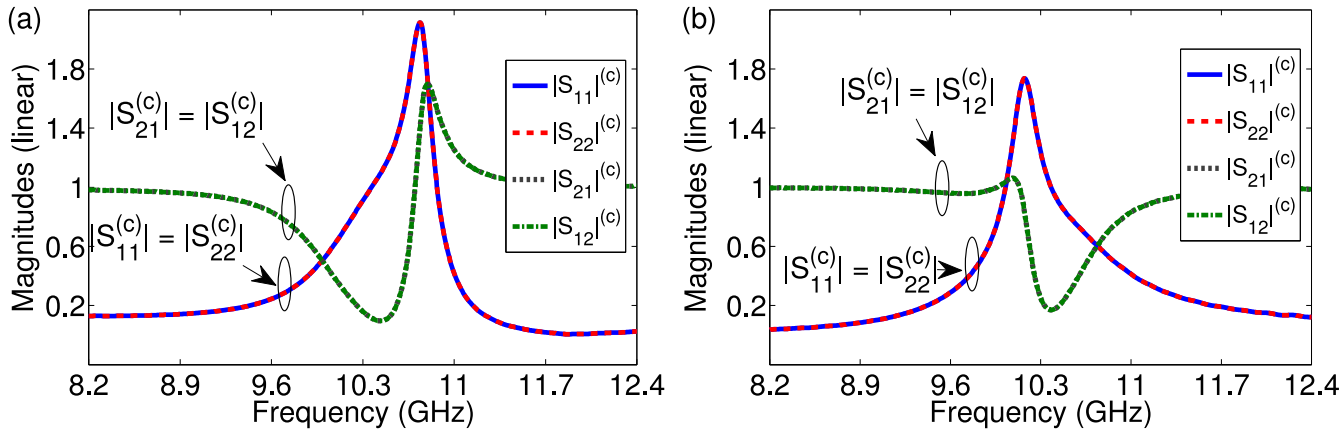

Fig. 7. Extracted $\left|S_{11}^{(c)}\right|,\left|S_{21}^{(c)}\right|,\left|S_{12}^{(c)}\right|$, and $\left|S_{22}^{(c)}\right|$ for cascaded (a) two $\mathrm{MM}_{7}$ slabs $\left(\delta_{s}=0.025\right)$ and (b) two $\mathrm{MM}_{11}$ slabs $\left(\delta_{s}=0.025\right)$.

and $S_{21}^{(c)}=S_{12}^{(c)}=1$. It is noted from Figs. 9(a) and 9 (b) that almost identical simulated and calculated $\left|S_{11}^{3 T}\right|,\left|S_{21}^{3 T}\right|,\left|S_{12}^{3 T}\right|$, and $\left|S_{22}^{3 T}\right|$ values for both cascaded MM slabs indicate negligible amount of coupling (weak coupling) between MM slabs. To verify this claim, $\left|S_{11}^{(c)}\right|,\left|S_{21}^{(c)}\right|$, 


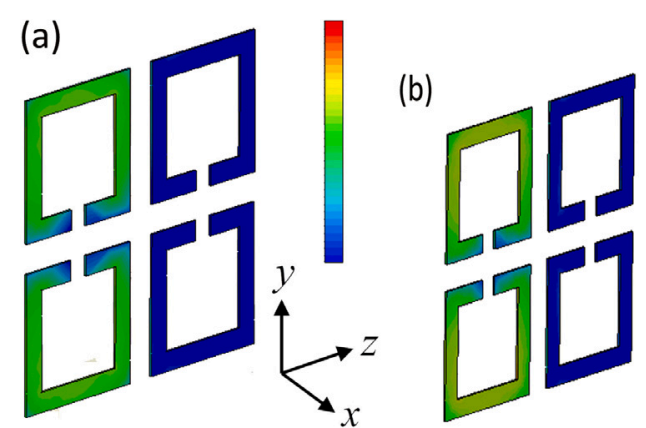

Fig. 8. Space distribution of the magnitudes of the magnetic field intensity for cascaded (a) $\mathrm{MM}_{7}$ slabs at their resonance frequencies $10.485 \mathrm{GHz}$ and (b) $\mathrm{MM}_{11}$ slabs at their resonance frequencies $10.371 \mathrm{GHz}$. Red color shows the highest value of $0 \mathrm{~dB} A / \mathrm{m}$ while blue color shows the lowest value of $-40 \mathrm{~dB} \mathrm{~A} / \mathrm{m}$. (For interpretation of the references to colour in this figure legend, the reader is referred to the web version of this article.)

$\left|S_{12}^{(c)}\right|$, and $\left|S_{22}^{(c)}\right|$ were extracted, as given in Figs. 10(a) and 10(b), respectively. It is noted that extracted $\left|S_{21}^{(c)}\right|$ (and $\left|S_{12}^{(c)}\right|$ ) and $\left|S_{11}^{(c)}\right|$ (and $\left.\left|S_{22}^{(c)}\right|\right)$, respectively, were almost unity and zero over the entire band, thus verifying our claim. Also, the dependencies of the magnitude of the magnetic field intensity for the cascaded $\mathrm{MM}_{7}$ slabs $\left(\delta_{s}=0.025\right)$ and for the cascaded $\mathrm{MM}_{11}$ slabs $\left(\delta_{s}=0.025\right)$ at their resonance frequencies $(10.308 \mathrm{GHz}$ and $10.182 \mathrm{GHz}$, respectively) were analyzed as shown Figs. 11(a) and 11(b). It is evident from Fig. 11 that, similar to the dependence in Fig. 8, only pair in propagation direction is active for the cascaded MM slabs. On the other hand, Fig. 12(a) and (b) exhibit the dependencies of $\left|S_{11}^{(c)}\right|,\left|S_{21}^{(c)}\right|,\left|S_{12}^{(c)}\right|$, and $\left|S_{22}^{(c)}\right|$ for both cascaded MM slabs $\left(\delta_{s}=0.025\right)$ when the separation distance is reduced from $12 \mathrm{~mm}$ to $4 \mathrm{~mm}$. Non-unity extracted $\left|S_{21}^{(c)}\right|$ (or $\left|S_{12}^{(c)}\right|$ ) in Fig. 12 shows nonnegligible amount of coupling around the resonance region $\left(\left|S_{21}^{(c)}\right| \neq 1\right.$ or $\left.\left|S_{12}^{(c)}\right| \neq 1\right)$ between cascaded MM slabs in propagation direction. Furthermore, it is noticed from Figs. 7 and 12 that, as expected, the coupling degree between cascaded MM slabs when the separation distance between them is zero is higher than the coupling degree between cascaded MM slabs when the separation distance is $4 \mathrm{~mm}$. Other simulations were performed to assess the coupling and its degree between cascaded analyzed MM slabs with substrate loss $\delta_{s}=0.005$ and with different separation distances. For instance, Fig. 13(a) and (b) show the extracted $\left|S_{11}^{(c)}\right|,\left|S_{21}^{(c)}\right|,\left|S_{12}^{(c)}\right|$, and $\left|S_{22}^{(c)}\right|$ for both cascaded MM slabs $\left(\delta_{s}=0.005\right)$ for separation distance $4 \mathrm{~mm}$. From Figs. 12 and 13 , we note that when the loss behavior of the substrate increases, the coupling between MM slabs decrease accordingly.

\section{Uncertainty analysis}

As an indispensable part in many extraction or retrieval methods, an uncertainty analysis was performed to evaluate any possible errors in the application of our coupling analysis approach. In parallel with our recent paper [20], in this section we investigate the effect of presence of air gap between MM slabs because our approach in this paper is to evaluate any possible coupling or interference between closely or largely separated MM slabs in propagation direction. It is assumed that gap is introduced at the center (symmetrical with respect to $z$ direction) of the whole cascaded structure. For instance, for a separation distance $12 \mathrm{~mm}$ between MM slabs and gap region of $1 \mathrm{~mm}$, gap is assumed to be present between $8.54 \mathrm{~mm}(L+6 \mathrm{~mm})$ and $9.54 \mathrm{~mm}$ measured from the front/back face of the cascade connection of MM slabs.

Figs. 14 and 15 illustrate the effect of air gap between cascaded MM slabs $\left(\delta_{s}=0.025\right)$ in propagation direction for different separation distances. The following two key points are concluded from the dependencies in Figs. 14 and 15. First, presence of air gaps, although small $(0.05 \mathrm{~mm})$, between MM slabs considerably alters the dependencies of extracted $\left|S_{11}^{(c)}\right|,\left|S_{21}^{(c)}\right|,\left|S_{12}^{(c)}\right|$, and $\left|S_{22}^{(c)}\right|$ compared with the those for the no-gap case (e.g., see Figs. 7 and 14). Second, extracted $\left|S_{21}^{(c)}\right|$ and $\left|S_{12}^{(c)}\right|$ are identical even though the gap is present between the MM slabs. This is because the MM slabs considered in our analysis (in addition to the air medium and filling FR4 substrate) are reciprocal (transmission symmetric) and the composite structure composed of MM slabs, air medium, and FR4 substrate will be reciprocal, too [see (3) and (5)]. Third, the presence of air gaps in the cascaded MM slabs produces asymmetry in the direction of propagation, resulting in non-identical extracted $\left|S_{11}^{(c)}\right|$ and $\left|S_{22}^{(c)}\right|$. Fourth, we note that the discrepancy between extracted $\left|S_{11}^{(c)}\right|$ and $\left|S_{22}^{(c)}\right|$ introduced by the air gaps increases by separation distance and symmetry. Finally, we note from Figs. 10 and 15 that the presence of air gaps can result in non-zero extracted $\left|S_{11}^{(c)}\right|$ and $\left|S_{22}^{(c)}\right|$ and non-unity $\left|S_{21}^{(c)}\right|$ and $\left|S_{12}^{(c)}\right|$, which may be mistakenly interpreted that there exists coupling between cascaded slabs, especially for MM resonators' slabs (see Section 5).

\section{Experimental analysis}

\subsection{Experimental setup and its calibration}

Fig. 16 shows a photo of the measurement setup used to validate our analysis. This setup has a Keysight vector network analyzer (VNA) with model number N9918A operated to measure S-parameters at Xband. Its directivity is no less than $32 \mathrm{~dB}$ and its dynamic range is $90 \mathrm{~dB}$ over X-band. Phase-steady $3.5 \mathrm{~mm}$ coaxial cables of length $100 \mathrm{~cm}$ were connected between the VNA and X-band coaxial-towaveguide adapters. Two additional X-band waveguide sections with sufficient length (approximately two times the free-space wavelength at the mid-frequency) were placed between the measurement cell and the coaxial-to-waveguide adapters.

Because the ends of the rectangular waveguide section are genderless, and due to the relatively simpler implementation of its calibration standards, the thru-reflect-line (TRL) calibration procedure was applied to calibrate the measurement setup in Fig. 16 before starting the validation process of our proposed analysis. An X-band waveguide straight with length of $10.16 \mathrm{~mm}$ was utilized as a line standard, resulting in nearly $\pm 70^{\circ}$ phase value change from $90^{\circ}$ over full the entire X-band. A highly reflection metal termination was operated as the reflect standard for both ports. After performing the calibration, full verification was conducted before continuing with measurements. Toward this end, phase values and magnitudes of $S_{21}$ (or $S_{12}$ ) of the thru standard and the line standard, in addition to the magnitudes of $S_{11}$ (or $S_{22}$ ), were recorded. It was noted that phase values of $S_{21}$ (or $S_{12}$ ) were approximately less than $1^{\circ}$ at all frequencies in the X-band for the thru standard and approximately $-90^{\circ}$ at the mid-frequency of the $\mathrm{X}$-band for the line standard. It was also observed that the magnitudes of $S_{21}$ (or $S_{12}$ ) were not less than $-0.03 \mathrm{~dB}$ over the full X-band for both thru and line standards. Finally, it was also noticed that the magnitudes of $S_{11}$ (or $S_{22}$ ) were not greater than $-40 \mathrm{~dB}$ over the full X-band for the reflect standard. These results clearly validate the calibration's accuracy.

\subsection{Coupling between conventional isotropic slabs}

For validation of our approach for coupling analysis between cascaded slabs, S-parameters of a $L_{d}=3.85 \mathrm{~mm}$ PE sample (fully occupying the waveguide's cross section) and cascaded two identical PE samples, each having a $3.85 \mathrm{~mm}$ length in the direction of propagation were measured. A $9.40 \mathrm{~mm}$ waveguide straight was used as a measurement cell for S-parameter measurements. The magnitudes of the measured S-parameters are demonstrated in Fig. 17(a). It is noted from the dependencies in Fig. 17(a) that measured $\left|S_{11}^{(1)}\right|$ and $\left|S_{22}^{(1)}\right|$ and measured $\left|S_{21}^{(1)}\right|$ and $\left|S_{12}^{(1)}\right|$, respectively, overlap each other. A similar conclusion can be drawn for measured $\left|S_{11}^{(3)}\right|,\left|S_{22}^{(3)}\right|,\left|S_{21}^{(3)}\right|$ and $\left|S_{12}^{(3)}\right|$. 

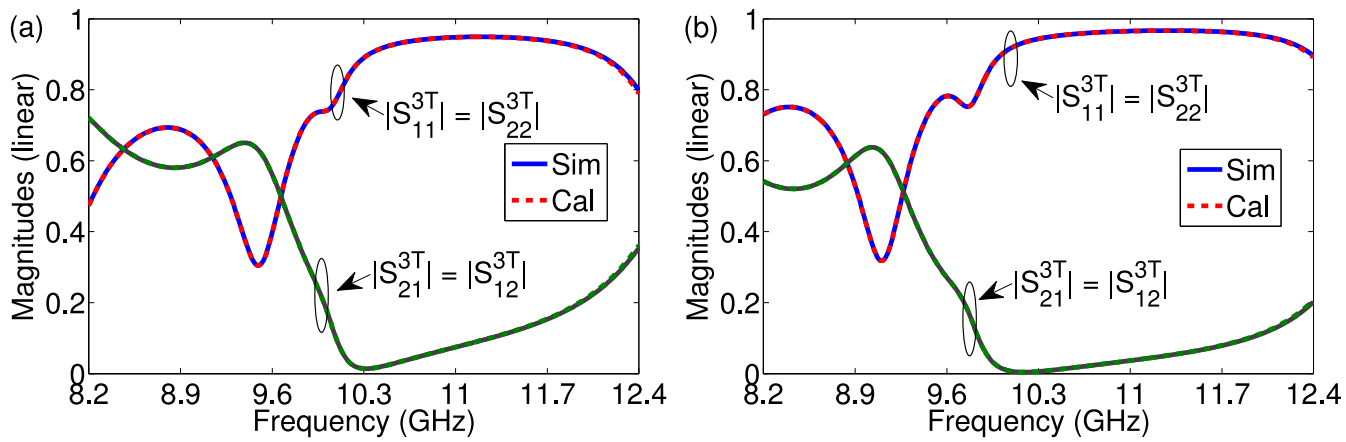

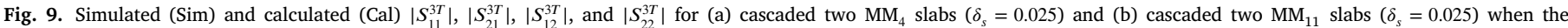

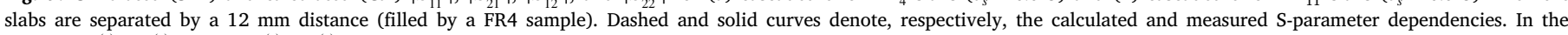
analysis, $S_{11}^{(c)}=S_{22}^{(c)}=0$ and $S_{21}^{(c)}=S_{12}^{(c)}=1$.
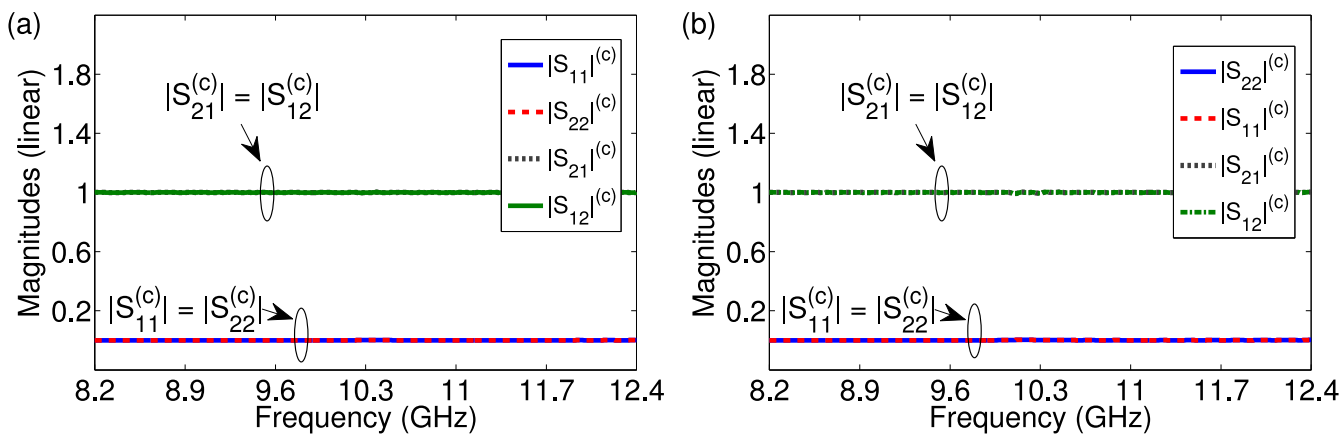

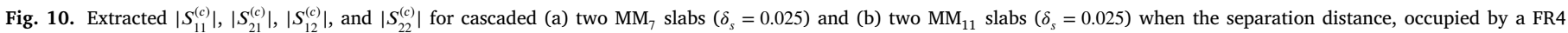
sample, between MM slabs is $12 \mathrm{~mm}$.
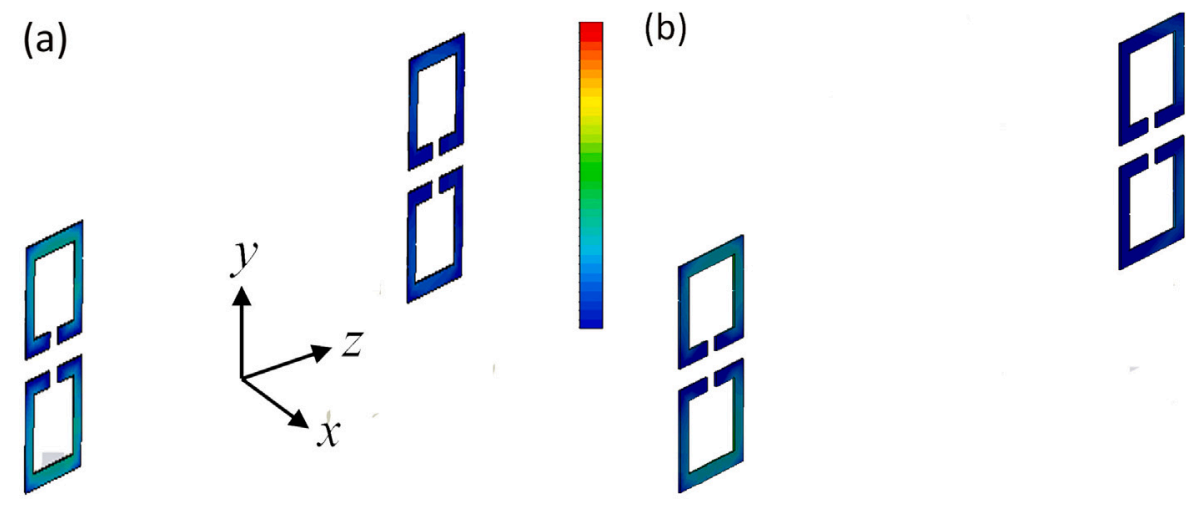

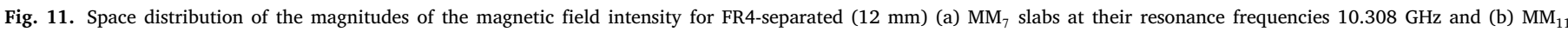

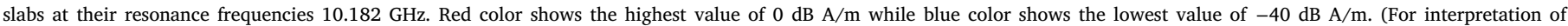
the references to colour in this figure legend, the reader is referred to the web version of this article.)
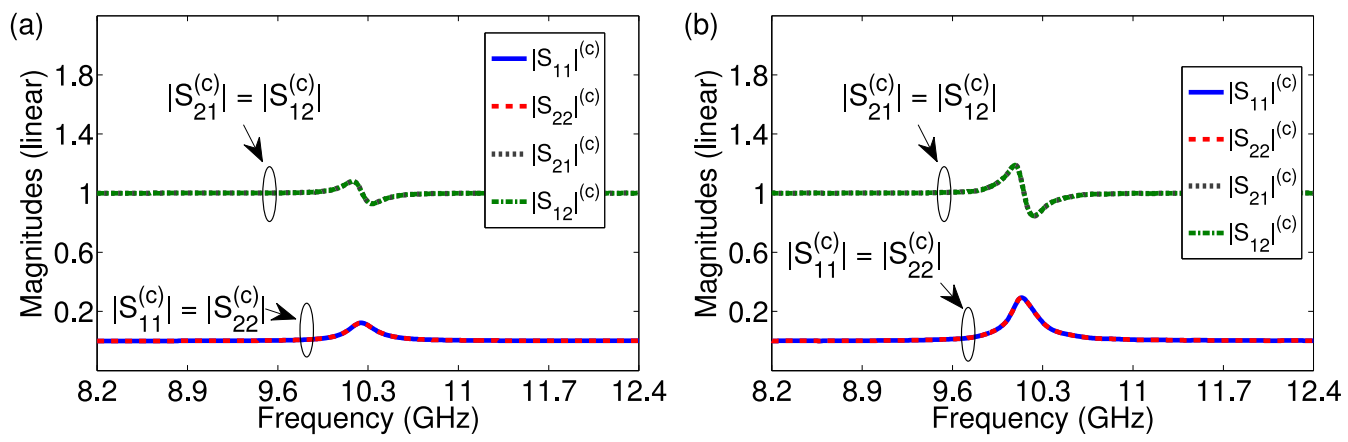

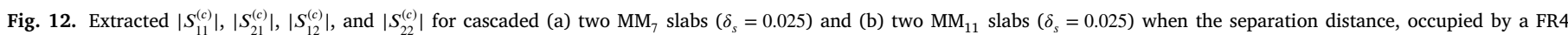
sample, between MM slabs is $4 \mathrm{~mm}$. 

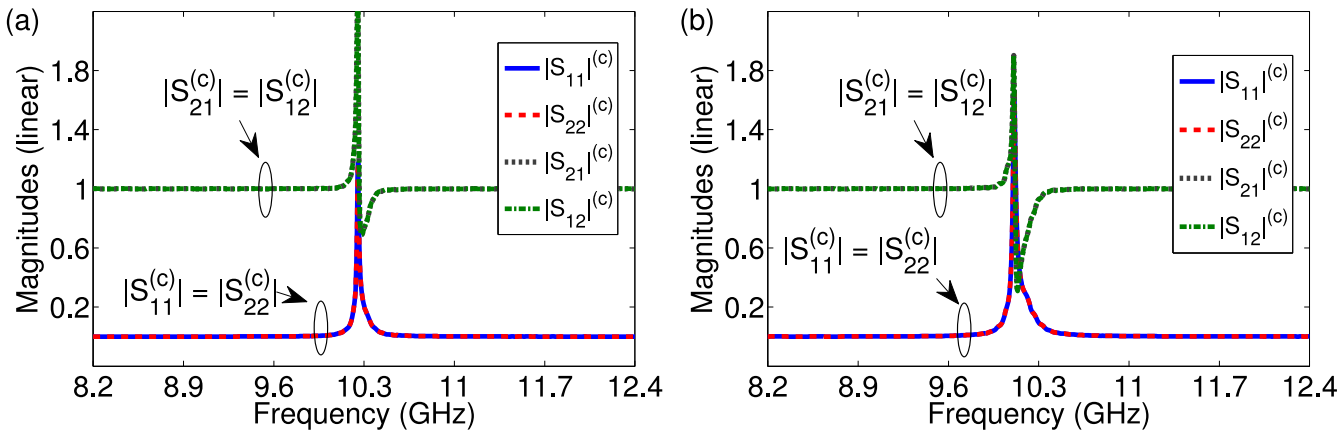

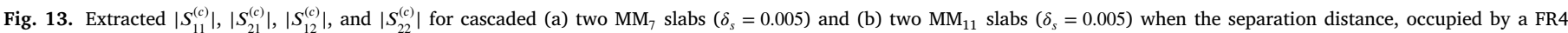
sample, between MM slabs is $4 \mathrm{~mm}$.
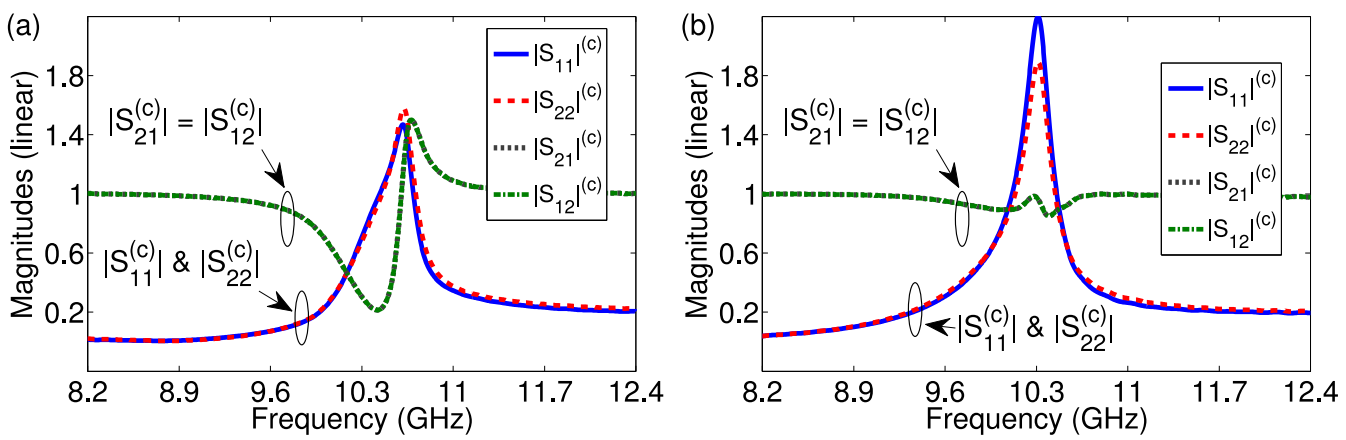

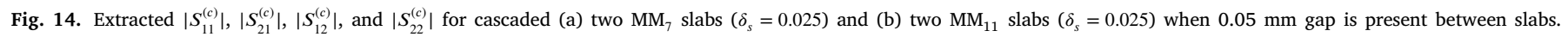
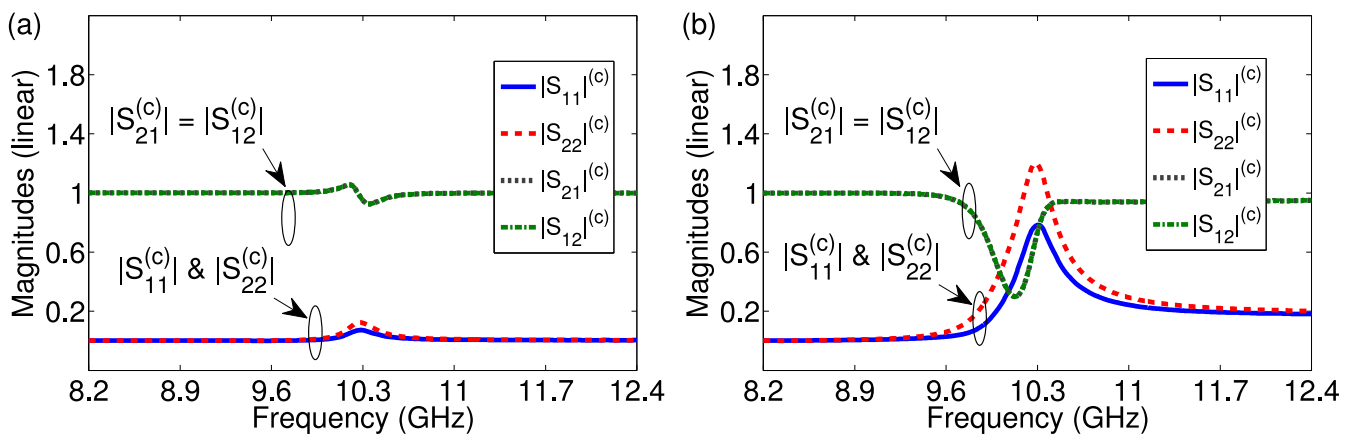

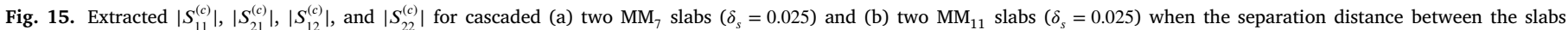
is $12 \mathrm{~mm}$ and when $0.05 \mathrm{~mm}$ gap is present at the center (symmetrical w.r.t. $z$ ) of whole cascaded structure.

This is because the PE material is a reflection-symmetric and reciprocal material. This further indicates that there is, as expected, no or negligible amount of coupling between the PE samples. To quantitatively validate this, $\left|S_{11}^{(c)}\right|,\left|S_{21}^{(c)}\right|,\left|S_{12}^{(c)}\right|$, and $\left|S_{22}^{(c)}\right|$ were extracted for two PE samples cascaded in the propagation direction, as shown in Fig. 17(b). It is seen from Fig. 17(b) that the measured $\left|S_{11}^{(c)}\right| \cong\left|S_{22}^{(c)}\right| \cong 0$ and $\left|S_{21}^{(c)}\right| \cong\left|S_{12}^{(c)}\right| \cong 1$, all validate our expectation.

\subsection{Coupling between MM slabs}

Next, we present coupling analysis of MM slabs in the direction of propagation. We fabricated two identical $(4 \times 7)$ MM slabs whose geometrical and electrical details are given in Section 3.1. The fabrication used conventional printed circuit board technique [20]. The thickness of the FR4 substrate is $1.5 \mathrm{~mm}$, which produces relatively slight additional air regions ( $0.77 \mathrm{~mm}$ on each side) inside the cross section along the $y$ direction. To reduce the effect of the air gap between substrates over the cross section of the guide, we inserted two unpatterned FR4 substrates with thicknesses of $0.77 \mathrm{~mm}$ over the $y z$ plane (and lengths of $2.54 \mathrm{~mm}$ ) right at the guide walls where the electric field intensity is minimum [20]. The periodicities in the $x$ and $y$ directions are approximately $u_{x}=3.266 \mathrm{~mm}$ and $u_{y}=2.54$ $\mathrm{mm}$, which are almost one-tenth of the free-space wavelength at the mid-frequency [42].

After construction of two identical $(4 \times 7) \mathrm{MM}$ slabs, our analysis was applied to monitor the coupling between these MM slabs. Fig. 18(a) and (b) illustrate the dependencies of the measured and calculated $\left|S_{11}^{3 T}\right|,\left|S_{21}^{3 T}\right|,\left|S_{12}^{3 T}\right|$, and $\left|S_{22}^{3 T}\right|$ for cascaded two $\mathrm{MM}_{7}$ slabs when there is no separation. In calculations, we set $S_{11}^{(c)}=S_{22}^{(c)}=0$ and $S_{21}^{(c)}=$ $S_{12}^{(c)}=1$. It is observed from Fig. 18 that when the MM slabs are connected directly, the measured and calculated $\left|S_{11}^{3 T}\right|,\left|S_{21}^{3 T}\right|,\left|S_{12}^{3 T}\right|$, and $\left|S_{22}^{3 T}\right|$ are considerably different, a manifestation of the presence of 


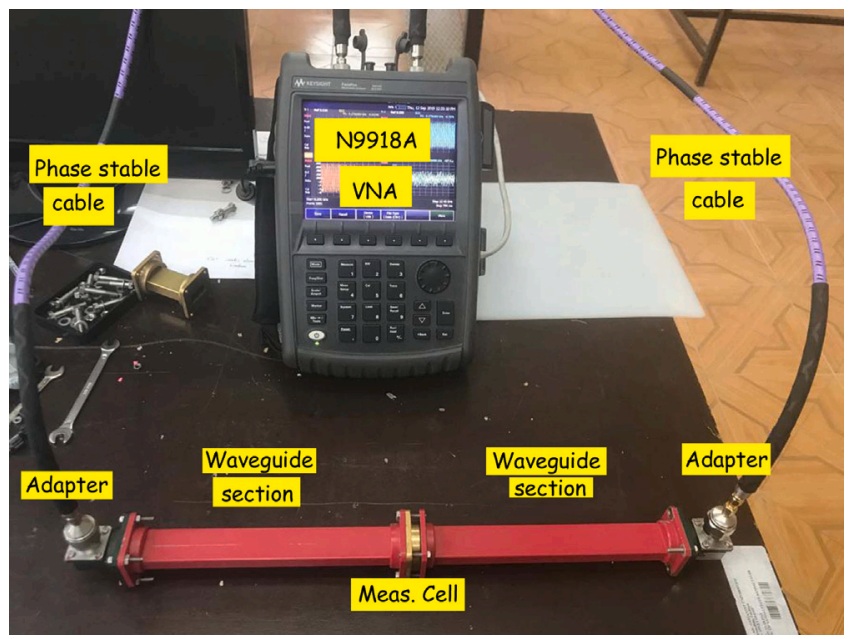

Fig. 16. The picture of the experimental setup used to validate our proposed analysis.

strong coupling between the cascaded MM slabs. We attribute the large deviation between the measured and simulated S-parameters above $11 \mathrm{GHz}$ in Fig. 18(a) and (b) to strong interaction between the MM slabs as compared with those at lower frequencies. To investigate the coupling phenomenon quantitatively, we draw, as shown in Fig. 19(a), the dependencies of $S_{11}^{(c)}, S_{22}^{(c)}, S_{21}^{(c)}$, and $S_{12}^{(c)}$ when there is no separation between the MM slabs. It is noted from Fig. 19(a) that $S_{21}^{(c)}$ and/or $S_{12}^{(c)}$ exceed the unity value around the resonance region of the MM slabs. This provides a validation that there is a strong interference (coupling) between directly connected MM slabs. Aside from this, we also monitored the effect of the separation distance between the MM slabs on the coupling. Fig. 19(b) illustrates extracted $\left|S_{11}^{(c)}\right|,\left|S_{21}^{(c)}\right|$, $\left|S_{12}^{(c)}\right|$, and $\left|S_{22}^{(c)}\right|$ when the separation distance between the MM slabs is $6 \mathrm{~mm}$. It is seen from 19(b) that the coupling between the MM slabs decreases considerably when the separation distance is $6 \mathrm{~mm}$. Here, for simplicity, the dependencies of the measured and calculated $\left|S_{11}^{3 T}\right|$, $\left|S_{21}^{3 T}\right|,\left|S_{12}^{3 T}\right|$, and $\left|S_{22}^{3 T}\right|$ are not shown when the slabs are separated by $6 \mathrm{~mm}$. As analyzed in Section 4, the discrepancy between $S_{11}^{(c)}$ and $S_{22}^{(c)}$ values in Fig. 19(b) arises from the presence of air gaps between the ends of the FR4 substrates and MM slabs.

It should be stressed that our proposed method in its current form is essentially applicable for coupling analysis in the direction of propagation, because the SFG technique and ABCD parameters are appropriate for analysis of wave propagation in a given direction [33]. In other words, the expressions in (6) and (7) are suitable for analyzing the coupling between adjacent MM slabs cascaded in the direction of propagation. Nonetheless, these expressions indirectly take into account the coupling in the transverse plane via change in $A_{1}, B_{1}, C_{1}, D_{1}, A_{2}, B_{2}$, $C_{2}, D_{2}, A_{\text {before }}, B_{\text {before }}, C_{\text {before }}, D_{\text {before }}, A_{\text {after }}, B_{\text {after }}, C_{\text {after }}, D_{\text {after }}$ due to a change in the periodicity (or coupling) in the transverse plane. As a future study, we will modify the proposed method to consider the coupling effect in the transverse plane of two-dimensional MMs (i.e., metasurfaces) [45].

\section{Conclusion}

The coupling or interference between closely or largely separated MM slabs has been examined. For this goal, the techniques relying on signal flow graph and $\mathrm{ABCD}$ parameters were applied. There are two main contributions of our study. As the first contribution, the calculated and simulated S-parameters of cascade connection of closely or largely separated MM slabs are compared to assess the degree of coupling between these slabs (qualitative analysis). The degree of coupling is closely associated with higher variation between the differences of the simulated and calculated S-parameters. As a second contribution, forward and backward S-parameters of the coupling network for a cascade connection of closely or largely separated MM slabs is extracted using the signal flow graph technique and $\mathrm{ABCD}$ parameters (quantitative analysis). Such an extraction indicates that if there is no coupling (such as cascade connection of conventional materials), magnitudes of the forward and backward transmission S-parameters for the coupling network are almost unity over the full frequency band, whereas the magnitudes of forward and backward reflection S-parameters are nearly zero. On the other hand, if there is coupling (such as cascade connection of MM slabs), the magnitudes of the forward (backward) transmission and reflection S-parameters digress, respectively, from unity and zero values especially for the resonance frequency region. S-parameter measurements of cascaded two MM slabs composed of SRRs were performed to validate the proposed analysis.

\section{CRediT authorship contribution statement}

Ugur Cem Hasar: Conceptualization, Methodology, Investigation, Writing - original draft. Yunus Kaya: Conceptualization, Writing review \& editing. Mucahit Izginli: Visualization, Writing - review \& editing. Hamdullah Ozturk: Visualization, Writing - review \& editing. Mehmet Ertugrul: Writing - review \& editing, Supervision. Omar M. Ramahi: Writing - review \& editing, Supervision.

\section{Declaration of competing interest}

The authors declare that they have no known competing financial interests or personal relationships that could have appeared to influence the work reported in this paper.
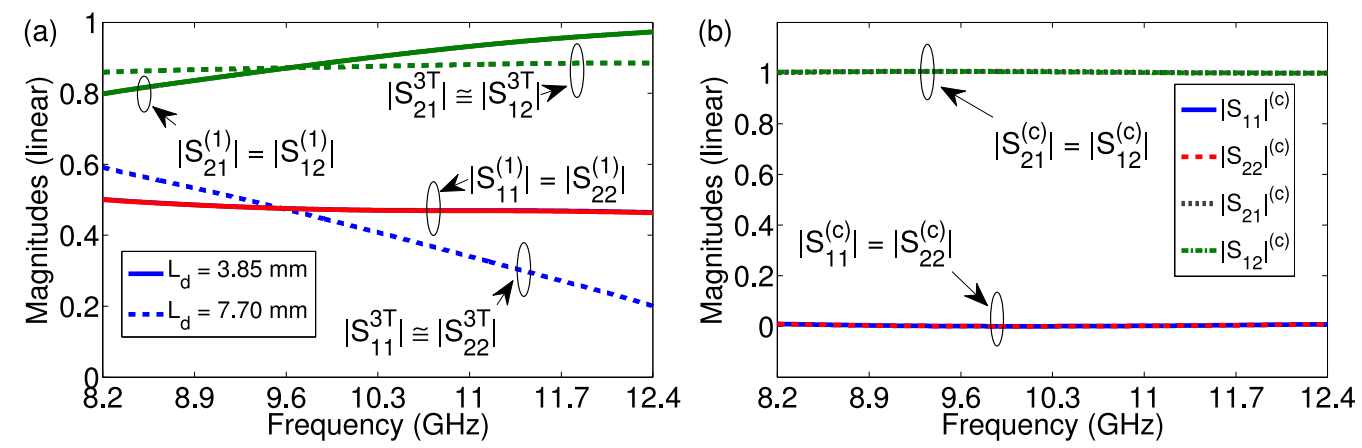

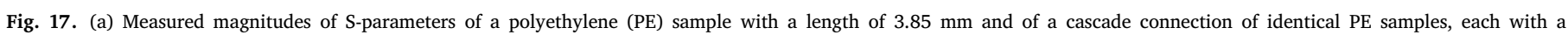
length of $3.85 \mathrm{~mm}$, and (b) extracted $\left|S_{11}^{(c)}\right|,\left|S_{21}^{(c)}\right|,\left|S_{12}^{(c)}\right|$, and $\left|S_{22}^{(c)}\right|$ for two PE samples cascaded in the direction of propagation. 

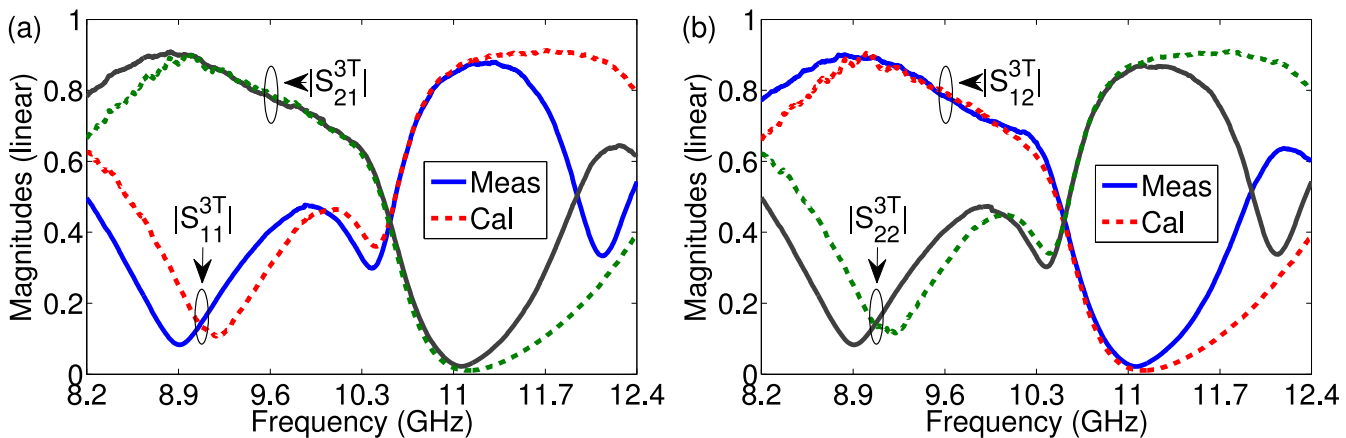

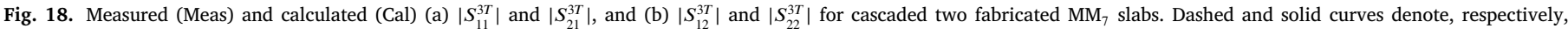
the calculated and measured S-parameter dependencies. In the analysis, $S_{11}^{(c)}=S_{22}^{(c)}=0$ and $S_{21}^{(c)}=S_{12}^{(c)}=1$.
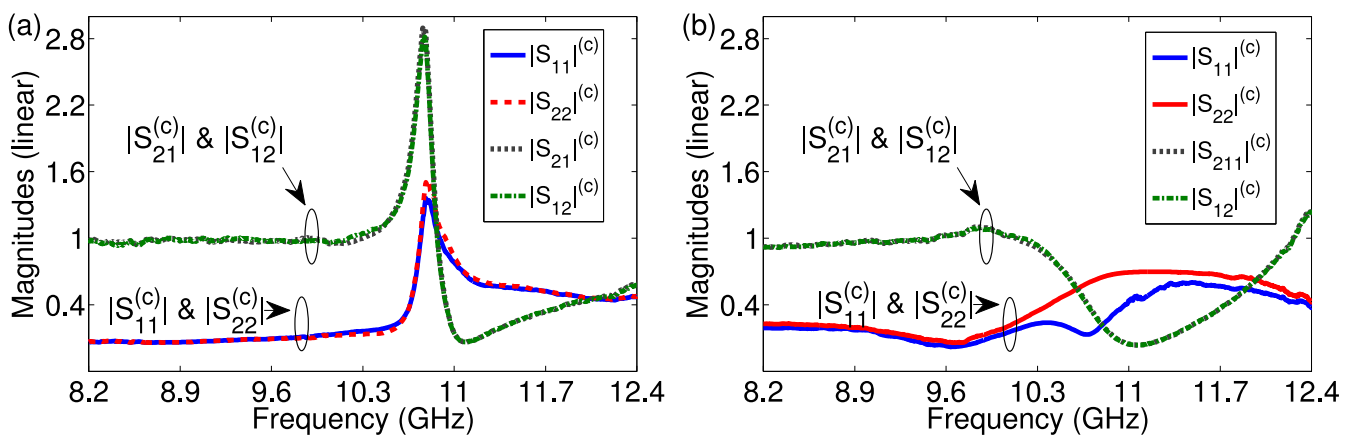

Fig. 19. Extracted $\left|S_{11}^{(c)}\right|,\left|S_{21}^{(c)}\right|,\left|S_{12}^{(c)}\right|$, and $\left|S_{22}^{(c)}\right|$ for cascaded two $\mathrm{MM}_{7}$ slabs with (a) no separation layer and (b) $6.0 \mathrm{~mm}$ long separation layer of FR4 substrate.

\section{References}

[1] R.A. Shelby, D.R. Smith, S. Shultz, Experimental verification of a negative index of refraction, Science 292 (5514) (2001) 77-79.

[2] J.B. Pendry, Negative refraction makes a perfect lens, Phys. Rev. Lett. 85 (2000) 3966.

[3] D. Schurig, J.J. Mock, B.J. Justice, S.A. Cummer, J.B. Pendry, A.F. Starr, D.R. Smith, Metamaterial electromagnetic cloak at microwave frequencies, Science 314 (5801) (2006) 977-980.

[4] F. Dincer, M. Karaaslan, E. Unal, C. Sabah, Dual-band polarization independent metamaterial absorber based on omega resoanator and octastar strip configuration, Prog. Electromagn. Res. 141 (2013) 219-231.

[5] E. Ekmekci, A.C. Strikwerda, K. Fan, G. Keiser, X. Zhang, G. Turhan-Sayan, R.D. Averitt, Frequency tunable terahertz metamaterials using broadside coupled split-ring resonators, Phys. Rev. B 83 (2011) 193103.

[6] R. Melik, E. Unal, N.K. Perkgoz, C. Puttlitz, H.V. Demir, Metamaterial based telemetric strain sensing in different materials, Opt. Express 18 (5) (2010) 5000-5007.

[7] R. Marqués, F. Medina, R. Rafii-El-Idrissi, Role of bianisotropy in negative permeability and left-handed metamaterials, Phys. Rev. B 65 (2002) 144440.

[8] J.B. Pendry, A.J. Holden, D.J. Robbins, W.J. Stewart, Low frequency plasmons in thin-wire structures, J. Phys.: Condens. Matter 10 (22) (1998) 4785-4809.

[9] A. Alù, First-principles homogenization theory for periodic metamaterials, Phys. Rev. B 84 (2011) 075153.

[10] D.R. Smith, S. Schultz, P. Markoš, C.M. Soukoulis, Determination of effective permittivity and permeability of metamaterials from reflection and transmission coefficients, Phys. Rev. B 65 (2002) 195104.

[11] X. Chen, T.M. Grzegorczyk, B.-I. Wu, J. Pacheco, J.A. Kong, Robust method to retrieve the constitutive effective parameters of metamaterials, Phys. Rev. E 70 (2004) 016608

[12] D.R. Smith, D.C. Vier, T. Koschny, C.M. Soukoulis, Electromagnetic parameter retrieval from inhomogeneous metamaterials, Phys. Rev. E 71 (2005) 036617.

[13] X. Chen, B.-I. Wu, J.A. Kong, T.M. Grzegorczyk, Retrieval of the effective constitutive parameters of bianisotropic metamaterials, Phys. Rev. E 71 (2005) 046610 .

[14] H. Chen, J. Zhang, Y. Bai, Y. Luo, L. Ran, Q. Jiang, J.A. Kong, Experimental retrieval of the effective parameters of metamaterials based on a waveguide method, Opt. Express 14 (26) (2006) 12 944-12 949.

[15] C. Menzel, C. Rockstuhl, T. Paul, F. Lederer, T. Pertsch, Retrieving effective parameters for metamaterials at oblique incidence, Phys. Rev. B 77 (2008) 195328.
[16] Z. Li, K. Aydin, E. Ozbay, Determination of the effective constitutive parameters of bianisotropic metamaterials from reflection and transmission coefficients, Phys. Rev. E 79 (2009) 026610.

[17] S. Kim, E.F. Kuester, C.L. Holloway, A.D. Scher, J. Baker-Jarvis, Boundary effects on the determination of metamaterial parameters from normal incidence reflection and transmission measurements, IEEE Trans. Antennas Propagation 59 (6) (2011) 2226-2240.

[18] O. Luukkonen, S.I. Maslovski, S.A. Tretyakov, A stepwise Nicolson-Ross-Weirbased material parameter extraction method, IEEE Antennas Wirel. Propag. Lett. 10 (2011) 1295-1298.

[19] D. Cohen, R. Shavit, Bi-anisotropic metamaterials effective constitutive parameters extraction using oblique incidence s-parameters method, IEEE Trans. Antennas Propag. 63 (5) (2015) 2071-2078.

[20] U.C. Hasar, A. Muratoglu, M. Bute, J.J. Barroso, M. Ertugrul, Effective constitutive parameters retrieval method for bianisotropic metamaterials using waveguide measurements, IEEE Trans. Microw. Theory Tech. 65 (5) (2017) 1488-1497

[21] R.S. Penciu, K. Aydin, M. Kafesaki, T. Koschny, E. Ozbay, E.N. Economou, C.M. Soukoulis, Multi-gap individual and coupled split-ring resonator structures, Opt. Express 16 (22) (2008) 18131-18144.

[22] P. Gay-Balmaz, O.J.F. Martin, Electromagnetic resonances in individual and coupled split-ring resonators, J. Appl. Phys. 92 (5) (2002) 2929.

[23] N. Katsarakis, T. Koschny, M. Kafesaki, E.N. Economou, C.M. Soukoulis, Electric coupling to the magnetic resonance of split ring resonators, Appl. Phys. Lett. 84 (15) (2004) 2943

[24] J. García-García, F. Martín, J.D. Baena, R. Marqués, L. Jelinek, On the resonances and polarizabilities of split ring resonators, J. Appl. Phys. 98 (2005) 033103.

[25] E. Ekmekci, K. Topalli, T. Akin, G. Turhan-Sayan, A tunable multi-band metamaterial design suing micro-split SRR structures, Opt. Express 17 (18) (2009) 16046-16058.

[26] I. Sersic, M. Frimmer, E. Verhagen, A.F. Koenderink, Electric and magnetic dipole coupling in near-infrared split-ring metamaterial arrays, Phys. Rev. Lett. 103 (2009) 213902

[27] M. Lapine, D. Powell, M. Gorkunov, I. Shadrivov, R. Marqués, Y. Kivshar, Structural tunability in metamaterials, Appl. Phys. Lett. 95 (8) (2009) 084105.

[28] J. Wang, S. Qu, J. Zhang, H. Ma, Y. Yang, C. Gu, X. Wu, Z. Xu, A tunable lefthanded metamaterial based on modified broadside-coupled split-ring resonators, Prog. Electromagn. Res. Lett. 6 (2009) 35-45.

[29] D.A. Powell, M. Lapine, M.V. Gorkunov, I.V. Shadrivov, Y.S. Kivshar, Metamaterial tuning by manipulation of near-field interaction, Phys. Rev. B 82 (2010) 155128 . 
[30] D.R. Chowdhury, R. Singh, A.J. Taylor, H.T. Chen, Ultrafast manipulation of near field coupling between bright and dark modes in terahertz metamaterial, Appl. Phys. Lett. 102 (1) (2013) 011122.

[31] D.R. Chowdhury, A.K. Azad, W. Zhang, R. Singh, Near field coupling in passive and active terahertz metamaterial devices, IEEE Trans. THz Sci. Technol. 3 (6) (2013) 783-790.

[32] M. Bute, U.C. Hasar, Analyzing coupling effect of adjacent resonating metamaterial slabs, 2016 International Conference for Students on Applied Engineering (ICSAE), Newcastle upon Tyne, 2016, pp. 98-103.

[33] D.M. Pozar, Microwave Engineering, Wiley, West Sussex, England, 2011.

[34] U.C. Hasar, J.J. Barroso, Scattering parameter analysis of cascaded bi-anisotropic metamaterials, Opt. Commun. 348 (2015) 13-18.

[35] U.C. Hasar, A new microwave method for electrical characterization of low-loss materials, IEEE Microw. Wirel. Compon. Lett. 19 (12) (2009) 801-803.

[36] U.C. Hasar, Y. Kaya, J.J. Barroso, M. Ertugrul, Determination of reference-plane invariant, thickness-independent, and broadband constitutive parameters of thin materials, IEEE Trans. Microw. Theory Tech. 63 (7) (2015) 2313-2321.

[37] U.C. Hasar, M. Bute, J.J. Barroso, C. Sabah, Y. Kaya, M. Ertugrul, Power analysis of multilayer structures composed of conventional materials and bi-anisotropic metamaterial slabs, J. Opt. Soc. Amer. B 31 (5) (2014) 939-947.

[38] Z.-G. Dong, S.-Y. Lei, Q. Li, M.-X. Xu, H. Liu, T. Li, F.-M. Wang, S.-N. Zhu, Non-left-handed transmission and bianisotropic effect in a $\pi$-shaped metallic metamaterial, Phys. Rev. B 75 (2007) 075117
[39] Z. Li, R. Zhao, T. Koschny, M. Kafesaki, K.B. Alici, E. Colak, H. Caglayan, E. Ozbay, C.M. Soukoulis, Chiral metamaterials with negative refractive index based on four U split ring resonators, Appl. Phys. Lett. 97 (2010) 081901.

[40] C. Sabah, Microwave response of octagon-shaped parallel plates: Lowloss metamaterial, Opt. Commun. 285 (2012) 4549-4552.

[41] C. Sabah, F. Urbani, Experimental analysis of $\Lambda$-shaped magnetic resonator for mu-negative metamaterials, Opt. Commun. 294 (2013) 409-413.

[42] F. Trang, H. Rogalla, Z. Popović, Effective constitutive parameters of hightemperature superconducting split-ring resonator arrays, IEEE Trans. Appl. Supercond. 25 (1) (2015) 1-7.

[43] U.C. Hasar, Y. Kaya, H. Ozturk, M. Izginli, J.J. Barroso, O.M. Ramahi, M. Ertugrul, Complex permittivity and thickness evaluation of low-loss dielectrics from uncalibrated free-space time-domain measurements, IEEE Trans. Geosci. Remote Sens. (2021) (in press).

[44] U.C. Hasar, G. Ozturk, Y. Kaya, M. Ertugrul, Calibration-free time-domain freespace permittivity extraction technique, IEEE Trans. Antennas Propagat. (2021) (in press).

[45] F. Ding, A. Pors, S.I. Bozhevolnyi, Gradient metasurfaces: A review of fundamentals and applications, Rep. Progr. Phys. 81 (2) (2017) 026401. 